images to directly observe Au-NPs incorporated into the enzyme units. The reconstituted GOx was then assembled on a gold electrode. In the second method, the electrode was first decorated with FADfunctionalized Au-NPs, and this surface was then used to reconstitute the GOx enzyme. Microgravimetric quartz-crystal microbalance measurements indicate a $60 \%$ surface coverage for biomaterials prepared by both methods.

Cyclic voltammetry studies of the biomaterials prepared by both methods show the glucose electro-oxidation current increases with glucose concentration. Furthermore, the unimolecular electron transfer rate constant that is calculated from the anodic current-density plateau is seven times larger than that measured for native GOx when oxygen is used as the electron acceptor. This indicates an increase in the turnover number for the enzyme that can only be attributed to the use of the Au-NP as the electron-relaying functionality. Control experiments show that the functioning of the bioelectrocatalyst is not affected by the presence of oxygen (a potential electron scavenger) or citric acid (an oxidizable interferant).

GREG KHITROV

\section{"Scarring" Produced by Packing of Spherical Particles onto the Surface of a Sphere}

Packing spherical particles onto the curved surface of a sphere produces "scars," distinctive high-angle grain boundaries, that are not observed in the packing of spherical particles onto planar surfaces. These scars are observed above a critical system size, and the number of additional defects in these scars increases linearly with the size of the sphere. These findings address a conundrum that has puzzled scientists for almost a century, dating back to theoretical work by J.J. Thomson, who attempted to explain the periodic table of the elements by modeling atoms in terms of rigid shells of electrons. Researchers A. Bausch (Department of Physics, Technische Universität München), M. Bowick (Department of Physics, Syracuse University), and colleagues from Fundamenteel Onderzoek der Materie, Institute for Atomic and Molecular Physics (Amsterdam); the University of Massachusetts; Harvard University; Iowa State University; and Ames National Laboratories reported their experimental studies of packing phenomena in the March 14 issue of Science.

Although scientists have theorized and used computational methods to predict minimum energy arrangements for dense packing of repulsive particles on spherical surfaces, the researchers in the present study observed this packing experimentally. The "repulsive particles" were crosslinked polystyrene beads ( $1 \mu \mathrm{m}$ in diameter) packed by adsorption on the spherical surfaces of water droplets of various size (e.g., $12 \mu \mathrm{m}$ radius) formed in an oil bath. The arrangement of particles was imaged using an inverted microscope, and the lattice geometry was analyzed using Delaunay triangulation algorithms for spherical surfaces.

Whereas repulsive particles on a planar surface will form a simple lattice of triangles akin to the familiar billiard-ball packing, the distinctive scars observed by the researchers are necessary for translating dense packing onto a curved surface. The scientists also observed that these grainboundary scars on the spherical surface terminate within the crystal lattice. A similar phenomenon of grain boundaries ending within the crystal is not typical on flat surfaces, due to the prohibitively high associated strain. Instead, flat surfaces display grain boundaries that terminate at the boundary of the sample. Theoretically, those planar grain boundaries can be considered "infinite" in length, since they extend across the periodic lattice.

The findings of this study may hold implications for such diverse fields as nanotechnology and drug design. Fullerenes, certain viruses and bacteria, and other naturally spherical surfaces are expected to display scars similar to those observed in the model polystyrene-bead spherical systems imaged by the researchers. The researchers see the next step as understanding how they can take advantage of these scars where they occur in nature.

According to Bowick, "Although dislocations are excited at finite temperature in flat crystals, our work shows that that spatial curvature can drive the proliferation of unusual dislocation arrays (scars) even at zero temperature. Such unavoidable irregularity in the ground state is a signature of curved geometries which we expect to encounter in many natural systems."

EMILY JARVIS

\section{Electric Field Drives \\ Particles to Self-Assemble}

With a combination of electric fields and fluid mixtures, researchers I. Aranson, M. Sapozhnikov, Y. Tolmachev, and W. Kwok of Argonne National Laboratory have caused tiny spheres of bronze and other metals to self-assemble into crystalline patterns, honeycombs, pulsating rings, and two-lobed structures that whirl like tiny propellers. Such self-assembling behavior could be exploited to create next- generation nanostructures and micromechanical devices.

As reported in the March 21 issue of Physical Review Letters, Aranson and colleagues placed a quarter-teaspoon of $100 \mu \mathrm{m}$ bronze spheres between two transparent sheets coated with conducting material. Under high voltage, each bronze sphere acquires a charge from the bottom plate and is attracted to the upper sheet. The spheres reverse charge when they hit the upper sheet and are repelled back toward the lower sheet. As the process repeats 40 times per second, the bronze particles form a shimmering "gas" between the two plates. Groups of particles, responding to the electric field from the plates and from each other, tend to cluster together and coalesce into large, random groups.

Sapozhnikov, a postdoctoral researcher working under Aranson's supervision, then filled the electrostatic cell with various nonconducting fluids, including toluene and octane. The results were essentially random until he tried phenotole, a colorless, oily fluid used in medicines and dyes. At around $1000 \mathrm{~V}$, the particles began to form regular patterns. By varying the voltage, the spacing between the plates and the amount of conductive fluid in the mix, the researchers found they could create a regularly spaced array of crystals, honeycombs, and other forms.

The results then were reproduced with other dielectric liquids mixed with a small amount of ethanol to control the electrical conductivity of the solution.

"Particles interact with each other and create hydrodynamic forces in the liquid. These interactions create the patterns," Aranson said. "You can actually 'tune' the patterns by adding impurities to the liquid."

The particles also form rings that grow, absorb other clusters of particles, and then burst open. "They grow, they rotate, they do all kinds of crazy things," Aranson said. "The rotation, especially, is still not understood."

Influencing nanometer-sized particles to self-assemble into useful structures is one of the field's most difficult challenges. Selfassembly techniques are usually driven by thermodynamic forces, which dictate the type of complex pattern formation.

"This electrostatic method provides an additional way to control the self-assembly process," Aranson said.

\section{AFM Adapted to Measure Adhesion between Carbon Nanotubes and Polymers}

Researchers at the Georgia Institute of Technology and the National Aeronautics 
and Space Administration (NASA) use chemical force microscopy to produce detailed information about adhesion between single-walled carbon nanotubes (SWNTs) and molecules of polymers with different functional groups. Their goal is to combine carbon nanotubes with lightweight polymers to produce composite materials with properties attractive for use on future space vehicles.

"Our hypothesis is that the stronger the adhesive interaction between molecules and nanotubes, the more likely it is that the polymer will fully wet the nanotubes, break up aggregations of nanotubes, and form a mechanically sound composite," said Larry Bottomley, a professor in the Georgia Tech School of Chemistry and Biochemistry. "The intent is to come up with two or three chemical groups that will give us the strongest interaction, and then incorporate these onto polymers for further studies."

Instead of using atomic force microscopy to map a surface, the researchers used the cantilever beam and deflection measurement to study the adhesion force between alkanethiol molecules on the microscope tip and nanotubes on the surface. As reported on March 23 at the 225th American Chemical Society National Meeting in New Orleans, the researchers raised a surface composed of nanotube bundles until it contacted the tip. When the nanotubes on the surface contacted the alkanethiols on the tip, they adhered to it. When the surface was lowered, the adhesive force between nanotubes and polymer pulled the cantilever down.

"If there are no adhesive interactions between the tip and the sample surface, the cantilever tip just lets go cleanly when you lower the surface," Bottomley said. "If there is strong adhesive interaction, the adhesive interaction bends the cantilever down until the restoration force of the cantilever exceeds the adhesive force. That provides a direct measurement of the adhesion."

The adhesion forces they are measuring with this method are in the nano-Newton range. Instead of a three-dimensional map of the surface, the technique produces a force volume image showing adhesion force variations across a two-dimensional surface.

From that information, Bottomley and collaborators M.A. Poggi of Georgia Tech and P.T. Lillehei of NASA judge which polymers-and functional groups-provide the best adhesion to the nanotubes.

"We find dramatic differences in the adhesive interactions with subtle changes in the chemistry of the tip," Bottomley said. "You have the strongest interactions in the amine-terminated samples compared to the methyl-terminated, hydroxylterminated, and carboxyl-acid-terminated composites."

Carbon nanotubes tend to clump

\section{International Congress on Materials Science and Nanotechnologies (NANMAT 2003)}

\section{October 22-24, 2003 \\ University Foundation Building \\ Brussels, Belgium}

\section{ANNOUNCEMENT AND CALL FOR PAPERS}

The European Academy of Sciences is organizing an International Congress on Materials Science and Nanotechnology (NANMAT 2003) to be held in Brussels, Belgium. The purpose of the conference is to discuss the most advanced methods and technologies in materials science, including nanomaterials. The Congress, sponsored by the Government of Brussels and capital and private industries, represents a major international event with the participation of outstanding scientists working on the leading edge of materials sciences and nanotechnologies.

\section{Deadline for submission of papers: July 15, 2003}

For more information, please contact: Rene Krapez, European Academy of Sciences

$$
\text { Tel: (32) } 22346383 \text { • Fax: (32) } 22347911 \text { • eurasc@skynet.be }
$$

\section{http:// www.crmus.org}

together into bundles, which can pose problems in composite manufacture. If the polymer does not interact with or "wet" the nanotubes individually, the result is a mechanical defect that will weaken the resulting composite, the researchers said.

Bottomley said, "The real challenge is distributing the nanotubes throughout the polymer in a proper orientation."

\section{Silica-Reinforced Rubber Provides a Temperature-Resistant Mount}

L. Reuvekamp from the University of Twente has mixed silica and rubber to produce a tire with a low rolling resistance, which will result in reduced fuel use for vehicles. Under a grant through the Netherlands Organization for Scientific Research, Reuvekamp mixed silica and rubber under the influence of organosilane. Tire manufacturers normally use carbon black instead of silica to strengthen the rubber of car tires. The organosilane used by Reuvekamp and colleagues acted as a coupling agent. It binds to the surface of silica and rubber, linking together two substances that can scarcely be coupled otherwise.

The ideal coupling temperature was determined experimentally. A temperature of at least $130^{\circ} \mathrm{C}$ is needed for the coupling agent to react with silica. The reaction temperature cannot go above $150^{\circ} \mathrm{C}$ or the rubber will vulcanize and become too hard for further processing.

The researchers discovered that zinc oxide, which is an essential part of the final tire, disrupts the coupling. However, they found that zinc oxide could be added after the coupling reaction without an adverse effect.

The researchers said that silica grain size influences the rolling resistance, and silica split into minute particles reduced this the most. The researchers have applied for a patent.

FOR MORE RESEARCI NEWS ON MATERIALS SCIENCE...

... access the

Materials Research Society

Web site:

www.mrs.org/gateway/ matl_news.html 